\title{
Clopidogrel-Induced Neutropenia after Coronary Stenting: Is Cilostazol a Good Alternative?
}

\author{
Massimo Montalto, ${ }^{1,2}$ Italo Porto, ${ }^{1,2,3}$ Antonella Gallo, ${ }^{1,2}$ Claudia Camaioni,, 2 \\ Roberta Della Bona, ${ }^{1,2}$ Antonio Grieco, ${ }^{1,2}$ Filippo Crea, ${ }^{1,2}$ and Raffaele Landolfi, ${ }^{1,2}$ \\ ${ }^{1}$ Institute of Internal Medicine, Catholic University of Rome, 00168 Rome, Italy \\ ${ }^{2}$ Institute of Cardiology, Catholic University of Rome, 00168 Rome, Italy \\ ${ }^{3}$ Department of Cardiovascular Medicine, Catholic University of Rome, Largo A. Gemelli 1, 00168 Rome, Italy
}

Correspondence should be addressed to Italo Porto, i.porto@doctors.org.uk

Received 11 January 2011; Revised 21 April 2011; Accepted 4 June 2011

Academic Editor: Robert M. Schainfeld

Copyright (C) 2011 Massimo Montalto et al. This is an open access article distributed under the Creative Commons Attribution License, which permits unrestricted use, distribution, and reproduction in any medium, provided the original work is properly cited.

Dual antiplatelet therapy with aspirin plus thienopyridines has become the standard treatment of patients undergoing coronary stenting. Clopidogrel has mostly replaced the use of ticlopidine due to its more favourable adverse event profile. However, also the use of clopidogrel is not without side effects. Clopidogrel major adverse events are represented by marrow suppression, manifesting with aplastic anaemia, thrombocytopenia and neutropenia. When clopidogrel toxicity occurs, there are few and unsubstantiated alternative treatments and thus, in these cases, medical decisions may be very difficult. We report a case of clopidogrel-induced bone marrow toxicity manifesting with severe neutropenia in a patient treated with multiple coronary stents and provide suggestions for an alternative treatment.

\section{Introduction}

Dual antiplatelet therapy with aspirin plus clopidogrel has become the standard treatment of patients undergoing coronary stenting $[1,2]$. Clopidogrel exerts its antiplatelet effect by inhibiting the binding of adenosine diphosphate (ADP) to its receptor (P2Y12) and consequent ADP-mediated platelet activation [3]. Current guidelines of the European Society of Cardiology suggest starting clopidogrel therapy before any percutaneous coronary intervention (PCI) or when acute coronary syndrome (ACS) occurs [4]. Clopidogrel use is associated with an increased risk of bleeding and may cause haematological adverse effects, such as thrombotic thrombocytopenic purpura, haemolytic uremic syndrome, and bonemarrow suppression, manifesting with aplastic anaemia, thrombocytopenia [5], and neutropenia [6, 7]. Minor side effects are represented by diarrhea, vomiting, hepatocellular injury, and skin rash [8-10]. It must be stressed that all these side effects occur a very low rate $[4,11]$. The CAPRIE trial, which included 9.599 patients treated with clopidogrel, showed a low annual incidence $(0.05 \%)$ of severe neutropenia, considered as neutrophil count below $0.45 \times$ $10^{9} / \mathrm{L}[4]$. Nevertheless, it is thought that the actual incidence of clopidogrel myelotoxicity could be somewhat underestimated [7].

When clopidogrel toxicity occurs, little is known about the efficacy and safety of alternative treatments, and thus, in these cases, medical decisions may be very difficult.

We report a case of clopidogrel-induced bone-marrow toxicity manifesting with severe neutropenia in a patient treated with multiple coronary stents and provide suggestions for an alternative treatment.

\section{Case Report}

A 65-year old Caucasian male was admitted to the Department of Internal Medicine of our Hospital for the sudden onset of palpable purpura on lower extremities. The patient was free from previous cardiovascular events, with untreated hypertension and smoker status as risk factors. He had no allergic history and denied any animal bite and recent ingestions of new drugs or diet components. 
All haematological, microbiological, immunological, and allergic tests were normal. Skin biopsy revealed leukocytoclastic vasculitis; therefore, oral steroid therapy was initiated. The patient remained free from angina and dyspnoea; nevertheless, the ECG showed ischemic alterations in the lateral leads, and an echocardiogram showed hypokinesis of the septal, lateral, and anterior wall. Cardiac enzymes were within the normal range. Coronary angiogram, performed after administration of aspirin (100 mg) and of a $300 \mathrm{mg}$ loading dose of clopidogrel, showed a critical stenosis of the left anterior descending artery involving the origin of the first diagonal branch and a severe short stenosis of a large, dominant circumflex artery at the level of the first marginal branch. Percutaneous coronary intervention (PCI) was then undertaken, which, due to the complex nature of the stenosis, required implantation of two drug-eluting stents (DESs) (Xience V, Abbot, Temecula, Calif, USA) on both bifurcations. A total of $45 \mathrm{~mm}$ of everolimus-eluting stent was used.

After the procedure, clopidogrel $(75 \mathrm{mg} /$ day $)$ and aspirin $(100 \mathrm{mg} /$ day $)$ were prescribed as chronic treatment. Five weeks later, the patient came back to our Emergency Department complaining of fever and dyspnoea. Blood tests revealed severe leukopenia (white cells $1 \times 10^{3} / \mu \mathrm{L}$ ) with marked neutropenia (neutrophils 3\%), anaemia (haemoglobin $9.9 \mathrm{~g} / \mathrm{dL}$ ), and an important rise in inflammatory markers (C-reactive protein $122 \mathrm{mg} / \mathrm{dL}$ ). A chest computed tomography revealed multiple inflammatory infiltrates. Cytological evaluation of bone-marrow aspirate showed hypoplasia of the granulocytic series with blocked maturation to the stage of promyelocytes. Clopidogrel-induced neutropenia was suspected, and therapy was immediately discontinued. Application of the Naranjo Causality Scale classified the adverse drug reaction as probable [11]. Empiric antibiotic therapy with levofloxacin (500 $\mathrm{mg}$ twice daily) was started and continued for one week. Granulocyte colonystimulating factor (G-CSF) $(5-10 \mu / \mathrm{Kg} / \mathrm{die})$ was administered for 72 hours. In the following ten days, total leucocytes and neutrophils count increased and returned within the normal range.

In consideration of the perceived high risk of stent thrombosis, it was necessary to replace clopidogrel with another drug in order to ensure adequate platelet inhibition. Intravenous infusion of eptifibatide $(2 \mu \mathrm{g} / \mathrm{kg} / \mathrm{min})$ was added to aspirin [12]. Moreover, anticoagulation was started, initially with enoxaparin $(100 \mathrm{UI} / \mathrm{Kg}$ twice daily) and then with oral anticoagulants (dicumarol, titrated to obtain an International Normalised Ratio of 2-3). A week later, eptifibatide infusion was discontinued, and cilostazol $100 \mathrm{mg}$ twice daily was started. Cilostazol is an antiplatelet that selectively inhibits cyclic nucleotide phosphodiesterase type 3 (PDE III) [13] and is commonly used for the management of the peripheral arterial disease. In the following days, the clinical conditions of the patient progressively improved. Neutrophil count remained within normal range, and the patient was discharged after six days. One year after the start of the combination therapy with aspirin, cilostazol, and oral anticoagulants, our patient remained asymptomatic. Repeat angiogram was performed, which showed absence of restenosis or plaque progression. Additionally, optical coherence tomography (OCT) showed complete neointimal coverage of the four implanted stents [14] (Figure 1). Cilostazol was thus stopped. Three months after cilostazol withdrawal, the patient is still in good health.

\section{Discussion}

Severe neutropenia has been reported as a rare adverse effect of thienopyridines, usually occurring from four weeks to three months after the start of therapy [3]. Neutropenia is less frequent with clopidogrel than with ticlopidine, as the latter directly inhibits colony-forming unit (CFU-C) replication in the bone marrow in a dose-dependent manner [15]. In our patient, clopidogrel was considered the most likely causative agent responsible for this severe haematological injury, as after few days from its withdrawal and after only three days of G-CSF therapy, neutrophil count rose and remained within the normal range for the following months [11].

Having to choose a new antiplatelet drug, we excluded the potential usage of another P2Y12 inhibitor due to the inherent risk of hematologic toxicity linked to drugs belonging to the same family. Ticlopidine was felt to be unsuitable for our patient due the well-known risk of bone-marrow complications reported for this drug $[3,16]$, and we also excluded the newly introduced P2Y12 inhibitor prasugrel for a possible cross-reaction. At the moment of the event, the nonthienopyridine ticagrelor had not been tested in large studies. Thus, we selected cilostazol.

Our choice was influenced by the favourable pharmacodynamic characteristics of the drug. Cilostazol is a PDEIII isozyme selective inhibitor which potently inhibits the activities of PDEIIIA, the cardiovascular PDEIII subtype, thus increasing the cyclic AMP (cAMP) content of human platelets. Additionally, cilostazol has another important pharmacological property: the blockage of platelet adenosine uptake. Intracellular cAMP is degraded via several PDE and synthesized by adenylate cyclase (AC). AC activity in turn is controlled by stimulatory (Gs) and inhibitory (Gi) G-proteins. Adenosine, either from cellular metabolism or extracellular sources, activates Gs via A2-receptors and Gi via A1-receptors. Platelets and vascular cells that on their surface expose A2-receptors, thus, after inhibition of adenosine uptake, increase their cAMP cytoplasmatic levels. Therefore, cilostazol increases cAMP levels in platelets thanks to both PDE inhibition and blockage of adenosine uptake [13].

The most commonly reported side effects for cilostazol in clinical trials are those related to the vasodilator effect of the drug, namely, headache (primarily responsible for the suspension of the treatment) dizziness, and peripheral edema. Gastrointestinal symptoms such as diarrhea are also common. Cardiovascular adverse events consist of palpitations, arrhythmias, and ventricular extrasystoles [17]. One hundred mg twice daily is the recommended dose.

In 2007, Schäfer et al. reported the occurrence of severe neutropenia under clopidogrel treatment three weeks after coronary stenting in a 65-year-old woman [18]. Since this patient was considered at very high risk of stent thrombosis, 


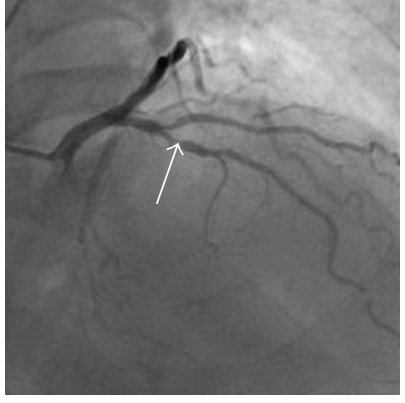

(a)

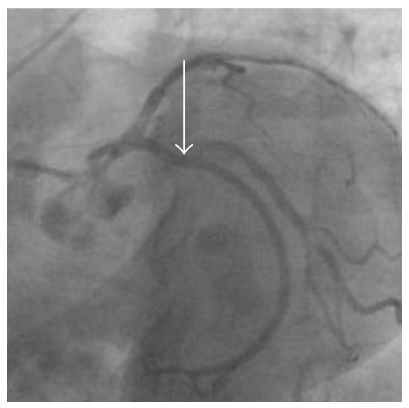

(e)

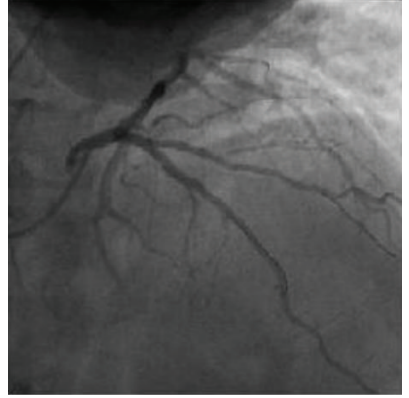

(b)

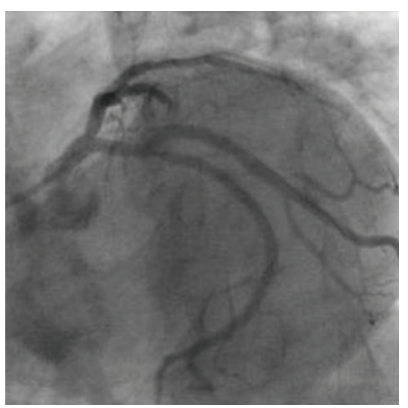

(f)

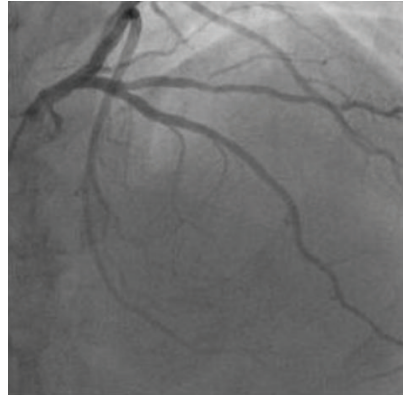

(c)

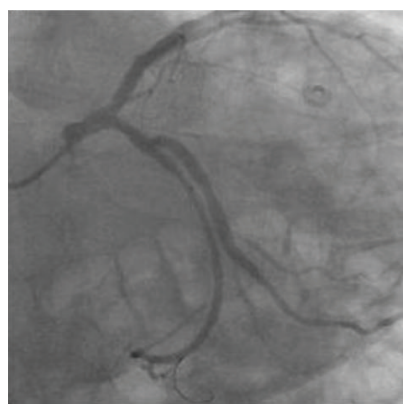

(g)
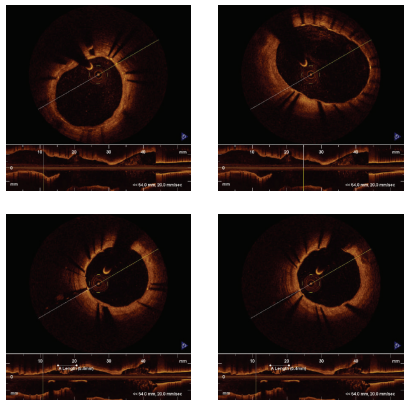

(d)
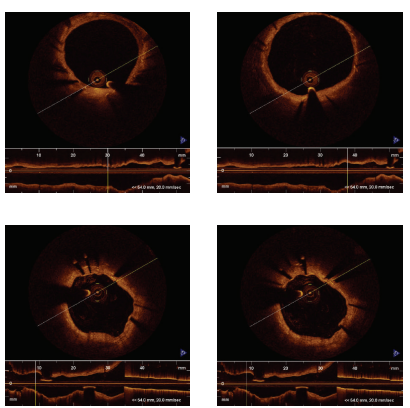

(h)

FIGURE 1: Upper panel shows the left coronary angiogram in the standard right anterior oblique cranial view. (a) At baseline, anterior descending artery (LAD) demonstrates a critical stenosis involving the origin of the first diagonal branch. (b) After two stents implantation in a "t and small protrusion"(TAP) fashion, good angiographic result can be seen. (c) One year angiographic followup attests that good result is maintained. (d) optical coherence tomography (OCT) analysis of LAD and diagonal branch (4 representative slices) at one year shows good neointimal coverage of the stent struts, without "malapposition". Lower panel shows the left coronary angiogram in the standard left anterior oblique view. (e) At baseline, critical stenosis of the circumflex artery (LCX) at the level of the first marginal branch is visible. (f) After two stents implantation in a "culotte" fashion, good angiographic result can be seen. (g) One year angiographic followup testifies the maintenance of good angiographic result. (h) optical coherence tomography (OCT) analysis of LAD and diagonal branch (4 representative slices) at one year shows good neointimal coverage of the stent struts, without "malapposition".

association treatment with aspirin $(150 \mathrm{mg} / \mathrm{d})$ and dipyridamole $(200 \mathrm{mg} / \mathrm{bid})$ was started. Also, oral anticoagulant therapy was added, because impaired left ventricular function was present [19]. Available followup, however, was only two months.

In our case, similar to Schäfer's team, we opted for anticoagulation $[18,19]$, since left ventricular function was reduced; nevertheless, we introduced cilostazol instead of dipyridamole.

Our preference of cilostazol was conditioned by two factors: (1) cilostazol inhibits platelets more effectively because its action involves with two mechanisms (PDE inhibition and blockage of adenosine uptake); (2) on clinical grounds, dipyridamole has never been tested as a part of antiplatelet therapy after PCI with DES, whereas cilostazol has been used in several studies.

A large meta-analysis published in 2008 reviewed 23 randomized clinical trials (RCTs) for a total of 5428 patients. Coprimary end points were angiographic restenosis and repeat revascularization. Results showed the effectiveness of cilostazol in reducing restenosis rate and repeat revascularization after PCI with DES; furthermore, cilostazol also appeared to be safe, with no significant increase in the risk of stent thrombosis or bleeding [20].
Cilostazol has been tested as addition to the usual dual antiplatelet therapy (DA) to reduce the risk of stent thrombosis. A Korean study (2009) investigated the effectiveness of the triple antiplatelet therapy (TA) (aspirin, clopidogrel, plus cilostazol) in diabetic patients undergoing coronary stent. 55 type II diabetic patients DES-treated were stratified to receive DA $(n=34)$ and TA $(n=21)$. Platelet aggregation was studied with adenosine diphosphate (ADP) stimulation, and then, the antiplatelet power was compared using light transmittance aggregometry between groups. Results showed that TA was more potent than the DA. These findings suggest that TA may be more effective in preventing thrombotic complications after DES implantation in type 2 diabetic patients [21]. Similar conclusions, regardless of diabetes, arise from Tamhane's meta-analysis that analyzed 10 RCTs ( $n=2,809$ patients) comparing TA with standard DA: antiplatelet therapy with cilostazol was associated with a significant reduction in angiographic restenosis [22]. Another meta-analysis, including 1457 post-PCI patients, studied not only the angiographic restenosis rate, but also the frequency of major adverse cardiac and/or cerebrovascular events (MACE/MACCE), stent thrombosis and bleeding in TA versus DA, after a median follow-up period of 6-9 months. Coprimary end points were not only angiographic restenosis, 
but also the rates of major adverse cardiac and/or cerebrovascular events (MACE/MACCE), stent thrombosis, and bleeding in TA versus DA. This careful analysis showed that cilostazol was effective in reducing angiographic restenosis without any significant benefit for MACE/MACCE rates [23]. The CILTON-T trial (960 DES-treated patients enrolled and randomized to receive DA or TA) shows more disappointing results: despite the greater reduction of platelet reactivity by addition of cilostazol to conventional DA therapy, TA did not show superiority in reducing cardiac death, nonfatal myocardial infarction, ischemic stroke, and target lesion revascularization [24]. However, TA appeared to have a beneficial clinical effect in an higher-risk population (patients with acute ST-segment elevation myocardial infarction (STEMI) undergoing PCI). A total of 4203 STEMI treated by DES were analyzed retrospectively. They received either DA $(n=2569)$ or TA $(n=1634)$. MACE after 8 months were significantly lower in the group with TA [25].

Of note, other studies tested the effectiveness and the safety of cilostazol as a substitute for clopidogrel in association with aspirin (the drug combination we used in our patient). A study on 280 patients showed that combination therapy with aspirin and cilostazol for the prevention of stent thrombosis (after only 1 month of aspirin, cilostazol, and clopidogrel combination treatment) was comparable or superior to aspirin and clopidogrel in diabetic patients undergoing DES implantation [26]. In particular, the risk of late stent thrombosis, in this complex population with high baseline risk, was not increased. In a larger population (689 patients), another team also obtained the same results although the patients were all treated with BMS [27]. More recently, a large, randomised Korean study in 1315 DEStreated patients proved the efficacy of cilostazol in the prevention of stent thrombosis. During the initial six months after PCI, patients were randomized to TA or DA (aspirin + clopidogrel); then, after this first period, all patients were randomized to DA with aspirin and cilostazol or DA with aspirin and clopidogrel [28]. Results were encouraging for cilostazol both in TA (association) and in DA (alternative) for the prevention of stent thrombosis.

As our strategy led to a successful long-term outcome for a difficult patient, we speculate that cilostazol might constitute an acceptable alternative treatment when thienopyridines are absolutely contraindicated, such as in cases of bone marrow toxicity. Further large-scale, randomized trials, however, are needed to fully elucidate this topic.

\section{References}

[1] I. Porto, S. Giubilato, G. L. De Maria, L. M. Biasucci, and F. Crea, "Platelet P2Y12 receptor inhibition by thienopyridines: status and future," Expert Opinion on Investigational Drugs, vol. 18, no. 9, pp. 1317-1332, 2009.

[2] Z. M. Chen, L. X. Jiang, Y. P. Chen et al., "Addition of clopidogrel to aspirin in 45852 patients with acute myocardial infarction: randomised placebo-controlled trial," The Lancet, vol. 366, no. 9497, pp. 1607-1621, 2005.
[3] P. J. Sharis, C. P. Cannon, and J. Loscalzo, "The antiplatelet effects of ticlopidine and clopidogrel," Annals of Internal Medicine, vol. 129, no. 5, pp. 394-405, 1998.

[4] W. Wijns, P. Kolh, N. Danchin et al., "Guidelines on myocardial revascularization: the task force on myocardial revascularization of the European Society of Cardiology (ESC) and the European Association for Cardio-Thoracic Surgery (EACTS)," European Heart Journal, vol. 31, no. 20, pp. 2501-2555, 2010.

[5] CAPRIE Seering Committee, "A randomised, blinded, trial of clopidogrel versus aspirin in patients at risk of ischaemic events (CAPRIE)," The Lancet, vol. 348, no. 9038, pp. 1329$1339,1996$.

[6] A. Zakarija, H. C. Kwaan, J. L. Moake et al., Kidney International, vol. 75, no. 112, pp. S20-S24, 2009.

[7] Z. A. Almsherqi, C. S. McLachlan, and S. M. Sharef, "Nonbleeding side effects of clopidogrel: have large multi-center clinical trials underestimated their incidence?" International Journal of Cardiology, vol. 117, no. 3, pp. 415-417, 2007.

[8] M. W. McCarthy and D. R. Kockler, "Clopidogrel-associated leukopenia," Annals of Pharmacotherapy, vol. 37, no. 2, pp. 216-219, 2003.

[9] R. K. Goyal, D. Srivastava, and K. D. Lessnau, "Clopidogrelinduced hepatocellular injury and cholestatic jaundice in an elderly patient: case report and review of the literature," Pharmacotherapy, vol. 29, no. 5, pp. 608-612, 2009.

[10] K. Makkar, R. L. Wilensky, M. B. Julien, H. C. Herrmann, and S. A. Spinler, "Rash with both clopidogrel and ticlopidine in two patients following percutaneous coronary intervention with drug-eluting stents," Annals of Pharmacotherapy, vol. 40, no. 6, pp. 1204-1207, 2006.

[11] C. A. Naranjo, U. Busto, E. M. Sellers et al., "A method for estimating the probability of adverse drug reactions," Clinical Pharmacology and Therapeutics, vol. 30, no. 2, pp. 239-245, 1981.

[12] E. Mahmud and A. Prasad, "Optimal antiplatelet therapy during percutaneous coronary interventions includes glycoprotein IIb/IIIa inhibitors: just eliminate the infusion," Journal of the American College of Cardiology, vol. 53, no. 10, pp. 846$848,2009$.

[13] K. Schrör, “The pharmacology of cilostazol," Diabetes, Obesity and Metabolism, vol. 4, supplement 2, pp. S14-S19, 2002.

[14] F. Prati, E. Regar, G. S. Mintz et al., "Expert review document on methodology, terminology, and clinical applications of optical coherence tomography: physical principles, methodology of image acquisition, and clinical application for assessment of coronary arteries and atherosclerosis," European Heart Journal, vol. 31, no. 4, pp. 401-415, 2010.

[15] K. Ono, K. Kurohara, M. Yoshihara, Y. Shimamoto, and M. Yamaguchi, "Agranulocytosis caused by ticlopidine and its mechanism," American Journal of Hematology, vol. 37, no. 4, pp. 239-242, 1991.

[16] J. O. Lokhandwala, P. J. M. Best, J. H. Butterfield et al., "Frequency of allergic or hematologic adverse reactions to ticlopidine among patients with allergic or hematologic adverse reactions to clopidogrel," Circulation, vol. 2, no. 4, pp. 348351, 2009.

[17] C. M. Pratt, "Analysis of the cilostazol safety database," The American journal of cardiology, vol. 87, no. 12A, pp. 28D-33D, 2001.

[18] A. Schäfer, M. Eigenthaler, and J. Bauersachs, "Severe neutropenia under clopidogrel treatment three weeks after coronary stenting," Thrombosis and Haemostasis, vol. 97, no. 2, pp. 317-318, 2007. 
[19] A. Schäfer, M. Eigenthaler, and J. Bauersachs, "Platelet activation in heart failure," Clinical Laboratory, vol. 50, no. 9-10, pp. 559-566, 2004.

[20] G. G. L. Biondi-Zoccai, M. Lotrionte, M. Anselmino et al., "Systematic review and meta-analysis of randomized clinical trials appraising the impact of cilostazol after percutaneous coronary intervention," American Heart Journal, vol. 155, no. 6, pp. 1081-1089, 2008.

[21] T. H. Yang, D. I. Kim, J. Y. Kim et al., "Comparison of triple anti-platelet therapy (aspirin, clopidogrel, and cilostazol) and double anti-platelet therapy (aspirin and clopidogrel) on platelet aggregation in type 2 diabetic patients undergoing drug-eluting stent implantation," Korean Circulation Journal, vol. 39, no. 11, pp. 462-466, 2009.

[22] U. Tamhane, P. Meier, S. Chetcuti et al., "Efficacy of cilostazol in reducing restenosis in patients undergoing contemporary stent based PCI: a meta-analysis of randomised controlled trials," EuroIntervention, vol. 5, no. 3, pp. 384-393, 2009.

[23] I. Singh, N. Shafiq, P. Pandhi et al., "Triple antiplatelet therapy vs. dual antiplatelet therapy in patients undergoing percutaneous coronary intervention: an evidence-based approach to answering a clinical query," British Journal of Clinical Pharmacology, vol. 68, no. 1, pp. 4-13, 2009.

[24] J. W. Suh, S. P. Lee, K. W. Park et al., "Multicenter randomized trial evaluating the efficacy of cilostazol on ischemic vascular complications after drug-eluting stent implantation for coronary heart disease: results of the CILON-T (influence of CILostazol-based triple antiplatelet therapy ON ischemic complication after drug-eluting stenT implantation) trial," Journal of the American College of Cardiology, vol. 18, no. 57, pp. 280-289, 2011.

[25] K. Y. Chen, S. W. Rha, Y. J. Li et al., "Triple versus dual antiplatelet therapy in patients with acute ST-segment elevation myocardial infarction undergoing primary percutaneous coronary intervention," Circulation, vol. 119, no. 25, pp. 32073214, 2009.

[26] Y. Ahn, H. J. Myung, W. J. Jeong et al., "Randomized comparison of cilostazol vs clopidogrel after drug-eluting stenting in diabetic patients - clilostazol for diabetic patients in drugeluting stent (CIDES) trial," Circulation Journal, vol. 72, no. 1, pp. 35-39, 2008.

[27] S. W. Lee, S. W. Park, M. K. Hong et al., "Comparison of cilostazol and clopidogrel after successful coronary stenting," American Journal of Cardiology, vol. 95, no. 7, pp. 859-862, 2005.

[28] D. S. Jeon, K. D. Yoo, C. S. Park et al., "The effect of cilostazol on stent thrombosis after drug-eluting stent implantation," Korean Circulation Journal, vol. 40, no. 1, pp. 10-15, 2010. 


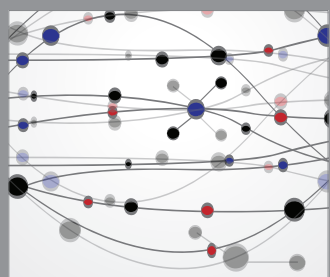

The Scientific World Journal
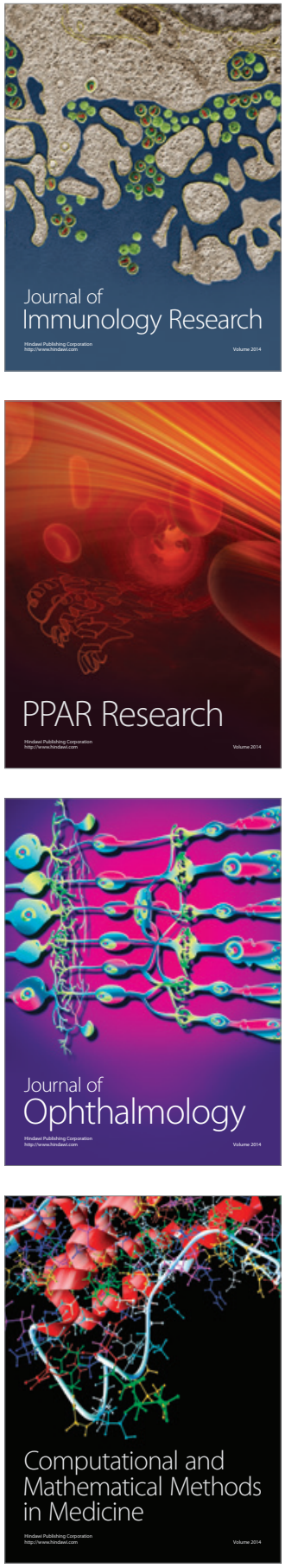

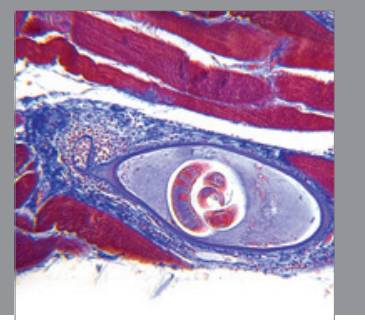

Gastroenterology

Research and Practice
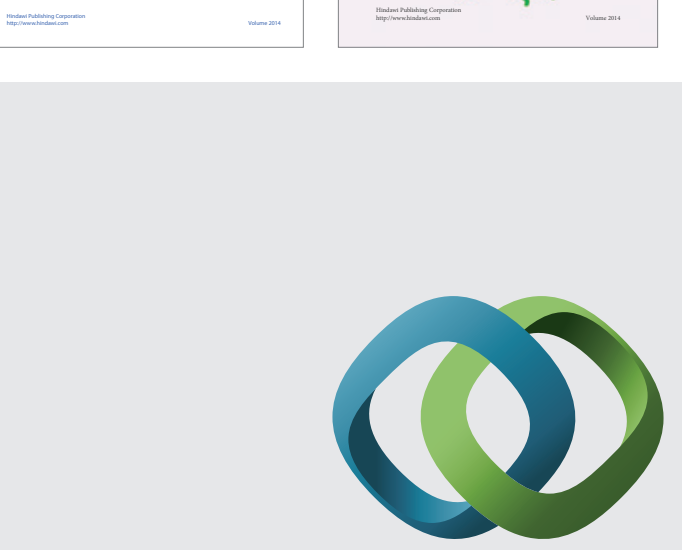

\section{Hindawi}

Submit your manuscripts at

http://www.hindawi.com
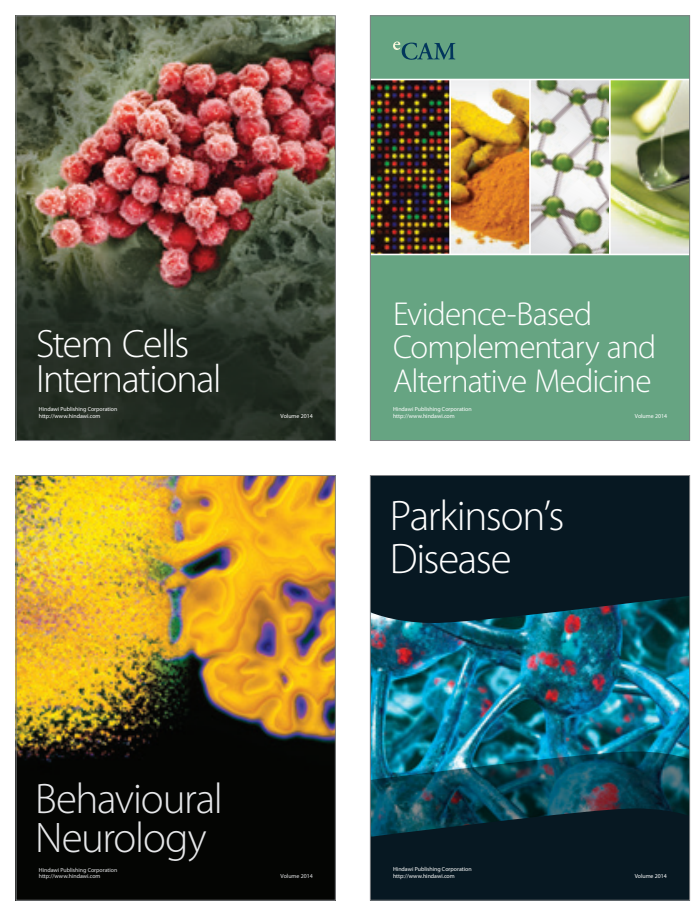

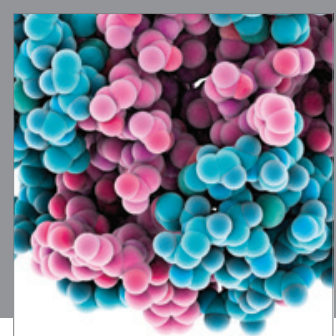

Journal of
Diabetes Research

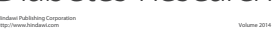

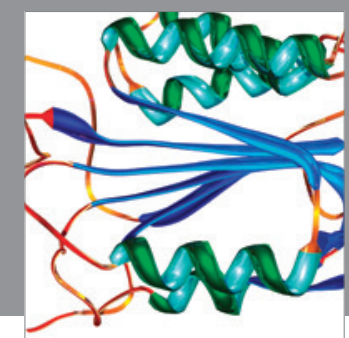

Disease Markers
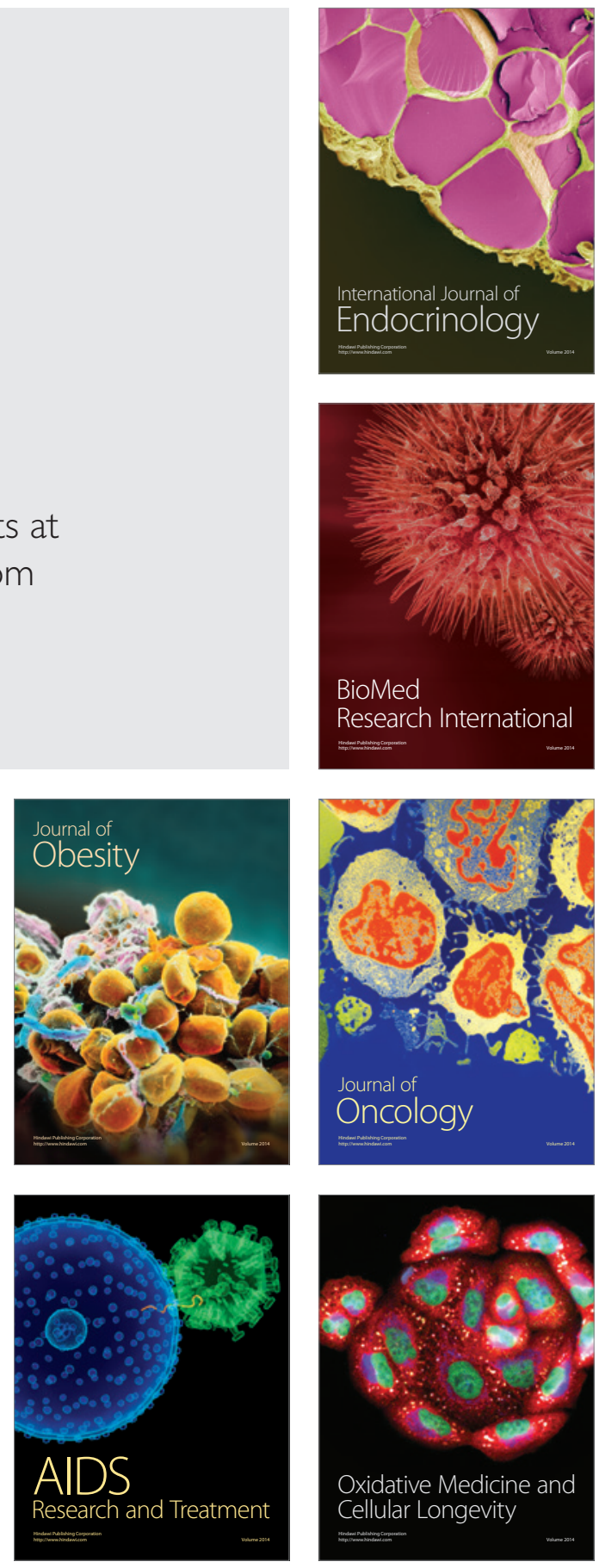